\title{
Multiscaling in Ising quantum chains with random Hilhorst-van Leeuwen perturbations
}

\author{
L. Turban \\ Laboratoire de Physique des Matériaux, UMR CNRS 7556, \\ Université Henri Poincaré, Nancy 1, \\ F-54506 Vandouvre lès Nancy Cedex, France
}

Received 5 July 2001

\begin{abstract}
We consider the influence on the surface critical behaviour of a quantum Ising chain of quenched random surface perturbations decaying as a power of the distance from the surface (random Hilhorst-van Leeuwen models). We study, analytically and numerically, the multiscaling behaviour of the surface magnetization and the surface energy density in the case of marginal perturbations.
\end{abstract}

Key words: Ising model, Hilhorst-van Leeuwen model, extended perturbations, randomness

PACS: 05.50.+q, 68.35.Rh

\section{Introduction}

In semi-infinite two-dimensional (2D) Ising models, extended perturbations of the coupling constants of the form $\Delta K_{l}=A l^{-\kappa}$, decaying as a power of the distance $l$ from a free surface, were first studied by Hilhorst and van Leeuwen $(\mathrm{HvL})$ at the begining of the 1980s [1]. Since then they have been the subject of continuous interest [2-15]. Similar extended perturbations associated with line defects in the bulk [16-18] or point defects [19-21] have also been considered (see Ref. [22] for a review).

Such extended perturbations are known to modify the local critical behaviour, for an arbitrarily small value of the perturbation amplitude $A$, as soon as the decay exponent $\kappa$ is sufficiently small. Analytical results for a surface defect in the $2 \mathrm{D}$ Ising model $[1,6]$ show that with a temperature-like perturbation (the case considered later) marginal behaviour is obtained when $\kappa=1$. Then the 
surface exponents vary continuously with the perturbation amplitude $A$ and the surface transition becomes first order when $A$ is greater than a critical value $A_{\mathrm{c}}$. When $\kappa<1$, the surface properties display essential singularities when $A<0$ and the transition is first order when $A>0$.

This rich critical behaviour has been explained independently by Cordery and Burkhardt $[2,3]$ through scaling considerations. Under a change of the length scale $l^{\prime}=l / b$, the perturbation transforms as

$$
\left[\Delta K_{l}\right]^{\prime}=\frac{A^{\prime}}{l^{\prime \kappa}}=b^{1 / \nu} \frac{A}{l^{\kappa}}
$$

where $\nu$ is the bulk correlation length exponent. Comparing both sides of the last equation, one obtains the following scaling behaviour for the perturbation amplitude:

$$
A^{\prime}=b^{-\kappa+1 / \nu} A
$$

Thus, for the 2D Ising model, $\kappa_{\mathrm{c}}=1 / \nu=1$ corresponds to the critical value of the decay exponent below which the extended perturbation becomes relevant.

An interesting aspect of these smoothly inhomogeneous models is that in 2D their correlation functions are covariant under conformal transformations when the perturbation is marginal. Thus gap-exponent relations are satisfied $[12,14,18-20]$ and conformal profiles are obtained in the strip geometry [15], provided the form of the perturbation is also properly transformed as first noticed in Ref. [12].

Recently, aperiodic [23] and random [24,25] versions of the HvL model have been studied for the quantum Ising chain in a transverse field, which corresponds to the extreme anisotropic limit [26] of the 2D classical Ising model and belongs to the same universality class.

In Ref. [24] random extended perturbations decaying towards a pure bulk system were considered with two types of quenched randomness.

In model \#1, the random couplings take the following form:

$$
J_{l}=J\left[1+a_{1} \frac{(-1)^{f_{l}}}{l^{\kappa}}\right], \quad f_{l}=\left\{\begin{array}{lll}
1 & \text { with probability } & 1 / 2 \\
0 & 1 / 2
\end{array} .\right.
$$

Thus the average perturbation $\left\langle J_{l}-J\right\rangle$ vanishes. 
In model \#2

$$
J_{l}=J\left[1+f_{l} a_{2}\right], \quad f_{l}=\left\{\begin{array}{lll}
1 & l^{-\kappa} \\
0 & \text { with probability } & 1-l^{-\kappa}
\end{array} .\right.
$$

Then the average coupling $\left\langle J_{l}\right\rangle=J\left(1+a_{2} l^{-\kappa}\right)$ has the same form as in the HvL model.

In both models the transverse fields are kept constant, $h_{l}=h$.

A perturbation expansion of the average free energy and scaling considerations allowed us to obtain a relevance criterion for both types of perturbations [24]. With model \#1, the first-order correction to the average free energy vanishes and the second-order correction leeds to the following scaling behaviour for the amplitude $a_{1}$ of a thermal perturbation:

$$
a_{1}^{\prime}=b^{1 / \nu-\kappa-1 / 2} a_{1}
$$

With model \#2, the scaling behaviour of the perturbation amplitude $a_{2}$ is governed by the non-vanishing first-order correction to the average free energy so that

$$
a_{2}^{\prime}=b^{1 / \nu-\kappa} a_{2}
$$

as for the non-random HvL model in (2). According to (5) and (6) marginal behaviour is obtained for $\kappa=1 / 2$ with model \#1 and $\kappa=1$ with model $\# 2$.

Analytical expressions for the finite-size scaling behaviour at criticality of the average and typical surface magnetization were obtained in Ref. [24], in the case of marginal and relevant perturbations, in agreement with the results of numerical simulations. We gave also some conjectured expressions for the surface energy exponents in the marginal case.

In the present work, we extend our previous study by considering the finite-size scaling behaviour of the average of the moments of the surface magnetization $\left\langle m_{\mathrm{s}}^{q}(L)\right\rangle$ and of the singular part of the surface energy $\left\langle e_{\mathrm{s}}^{q}(L)\right\rangle$ in the marginal situation. In Section 2, we briefly recall the techniques used to diagonalize the corresponding quantum Hamiltonians and to obtain the scaling behaviour of the surface magnetization and surface energy. Analytical expressions for the average of the moments of the surface magnetization and the surface energy are deduced from scaling considerations in Sections 3. The results are compared to numerical finite-size scaling data in Section 4 and their range of validity discussed in Section 5. 


\section{Free fermions techniques}

\subsection{Diagonal Hamiltonian}

We consider the semi-infinite transverse-field Ising model (TIM) with Hamiltonian

$$
\mathcal{H}=-\frac{1}{2} \sum_{l=1}^{\infty}\left(J_{l} \sigma_{l}^{z} \sigma_{l+1}^{z}+h_{l} \sigma_{l}^{x}\right)
$$

where $\sigma_{l}^{x}$ and $\sigma_{l}^{z}$ are Pauli matrices, $J_{l}$ is the random coupling and $h_{l}$ the transverse field.

The TIM Hamiltonian can be diagonalized using standard methods [27,28]. A Jordan-Wigner transformation [29] changes the original Hamiltonian (7) into a quadratic form in fermions which is diagonalized through a canonical transformation leading to

$$
\mathcal{H}=\sum_{q=1}^{L} \epsilon_{q}\left(\eta_{q}^{\dagger} \eta_{q}-\frac{1}{2}\right)
$$

for a chain with length $L$. The $\eta_{q}^{\dagger}\left(\eta_{q}\right)$ are fermion creation (annihilation) operators and the non-negative excitation energies $\epsilon_{q}$ satisfy the set of equations

$$
\begin{aligned}
& \epsilon_{q} \psi_{q}(l)=-h_{l} \phi_{q}(l)-J_{l} \phi_{q}(l+1) \\
& \epsilon_{q} \phi_{q}(l)=-J_{l-1} \psi_{q}(l-1)-h_{l} \psi_{q}(l)
\end{aligned}
$$

with the boundary conditions $J_{0}=J_{L}=0$. Introducing $2 L$-dimensional vectors $\boldsymbol{V}_{q}$ with components

$$
V_{q}(2 l-1)=-\phi_{q}(l), \quad V_{q}(2 l)=\psi_{q}(l)
$$


the relations (9) lead to an eigenvalue problem for the following tridiagonal matrix:

$$
\mathrm{T}=\left(\begin{array}{cccccc}
0 & h_{1} & & & & \\
h_{1} & 0 & J_{1} & & & \\
& J_{1} & 0 & h_{2} & & \\
& & \ddots & \ddots & \ddots & \\
& & & J_{l-1} & 0 & h_{L} \\
& & & & h_{L} & 0
\end{array}\right)
$$

When $\mathrm{T}$ is squared, odd and even components of $\boldsymbol{V}_{q}$ decouple and one obtains two separate eigenvalue problems for $\boldsymbol{\phi}$ and $\boldsymbol{\psi}$. The relations (9) are invariant under the transformation $\phi_{q} \rightarrow-\phi_{q}, \epsilon_{q} \rightarrow-\epsilon_{q}$ so that, changing $\phi_{q}$ into $-\phi_{q}$ in $\boldsymbol{V}_{q}$, one obtains the eigenvector of $\mathrm{T}$ with eigenvalue $-\epsilon_{q}$. Thus the physically relevant information is contained in that part of the spectrum of $\mathrm{T}$ with $\epsilon_{q} \geq 0$.

\subsection{Surface magnetization and surface energy}

The surface critical properties can be obtained through finite-size scaling at criticality, i.e., by working on a finite system with length $L$ and $J=h=1$.

The imaginary time spin-spin autocorrelation function is given by

$$
\mathcal{G}_{l}^{\sigma \sigma}(\tau)=\left\langle 0\left|\sigma_{l}^{z}(\tau) \sigma_{l}^{z}(0)\right| 0\right\rangle=\sum_{n}\left|\left\langle n\left|\sigma_{l}^{z}\right| 0\right\rangle\right|^{2} \exp \left[-\tau\left(E_{n}-E_{0}\right)\right]
$$

where $|0\rangle$ and $|n\rangle$ are the ground state and the $n$th excited state of $\mathcal{H}, E_{0}$ and $E_{n}$ the corresponding eigenvalues. With fixed boundary conditions, $\sigma_{L}^{z}= \pm 1$, at $l=L$, the ground state is degenerate and asymptotically, one obtains $\lim _{\tau \rightarrow \infty} \mathcal{G}_{l}^{\sigma \sigma}(\tau)=m_{l}^{2}$ where

$$
m_{l}=\left\langle\sigma\left|\sigma_{l}^{z}\right| 0\right\rangle
$$

is the local magnetization. It is given by an off-diagonal matrix element involving the first excited state with one fermionic excitation, $|\sigma\rangle=\eta_{1}^{\dagger}|0\rangle$, which is degenerate with the ground state. For the surface spin at $l=1$ a simple 
expression is obtained [5]:

$$
m_{\mathrm{s}}(L)=m_{1}=\phi_{1}(1)=\left[1+\sum_{l=1}^{L-1} \prod_{k=1}^{l}\left(\frac{h_{k}}{J_{k}}\right)^{2}\right]^{-1 / 2}
$$

This expression can be rewritten under the following form [24]:

$$
m_{\mathrm{s}}(L)=\bar{m}_{\mathrm{s}}^{\mathrm{d}}(L) \prod_{l=1}^{L-1}\left(\frac{J_{l}}{h_{l}}\right)
$$

where

$$
\bar{m}_{\mathrm{s}}^{\mathrm{d}}(L)=\left[1+\sum_{j=1}^{L-1} \prod_{k=L-j}^{L-1}\left(\frac{J_{k}}{h_{k}}\right)^{2}\right]^{-1 / 2}
$$

is the surface magnetization at $l=L-1$ on the dual chain with transverse fields $J_{l}$, couplings $h_{l}(l=0, L-1)$ and fixed boundary conditions $J_{0}=0$ at $l=0$.

The scaling dimension of the surface energy density can be obtained by considering the finite-size behaviour with free boundary conditions of the off-diagonal matrix element [30]

$$
e_{\mathrm{S}}(L)=\left\langle\varepsilon\left|\sigma_{1}^{x}\right| 0\right\rangle=\left(\epsilon_{2}-\epsilon_{1}\right) \phi_{1}(1) \phi_{2}(1),
$$

where $|\varepsilon\rangle=\eta_{1}^{\dagger} \eta_{2}^{\dagger}|0\rangle$ is the lowest eigenstate leading to a non-vanishing matrix element. This matrix element enters into the expression of the connected energy-energy surface autocorrelation function $\mathcal{G}_{1}^{\varepsilon \varepsilon}(\tau)$ and scales like the singular part of the surface energy density.

For the critical HvL model with a marginal decay of the perturbation, $h_{l}=1$, $J_{l}=1+a / l$, the product in (15) behaves asymptotically as $L^{a}$. When $L \gg 1$, the couplings on the right side of the chain are asymptotically unperturbed, thus one expects that $\bar{m}_{\mathrm{s}}^{\mathrm{d}}(L) \sim L^{-1 / 2}$ as for the homogeneous Ising model at the ordinary transition. This leads to the scaling behaviour:

$$
m_{\mathrm{s}}(L) \sim L^{-x_{\mathrm{m}}^{\mathrm{s}}}, \quad x_{\mathrm{m}}^{\mathrm{s}}=\frac{1}{2}-a, \quad a \leq \frac{1}{2}
$$

This expression cannot be valid beyond $a=1 / 2$ where the surface remains ordered at the bulk critical point $[6,5]$. The surface magnetization then displays a first order transition and $x_{\mathrm{m}}^{\mathrm{s}}=0$ when $a>a_{c}=1 / 2$. Thus our assumption 
concerning the behaviour of $\bar{m}_{\mathrm{s}}^{\mathrm{d}}(L)$ is wrong when $a>1 / 2$ and then one must have $\bar{m}_{\mathrm{s}}^{\mathrm{d}}(L) \sim L^{-a}$.

For the surface energy in Eq. (17), when $a \leq 1 / 2$ both $\phi_{1}(1)$ and $\phi_{2}(1)$ scale as $m_{\mathrm{s}}(L) \sim L^{-x_{\mathrm{m}}^{\mathrm{s}}}$. The excitations $\epsilon_{1}$ and $\epsilon_{2}$ scale as $L^{-1}$ leading to

$$
e_{\mathrm{s}}(L) \sim L^{-1} m_{\mathrm{s}}^{2}(L) \sim L^{-x_{\mathrm{e}}^{\mathrm{s}}}, \quad x_{\mathrm{e}}^{\mathrm{s}}=2(1-a), \quad a \leq \frac{1}{2},
$$

in the second-order regime. When $a>1 / 2$, the onset of surface order leads to a more complicated scaling behaviour. The first gap $\epsilon_{1}$ is associated with a localized state which reflects the surface behaviour. It scales as $L^{-2 a}$ while $\epsilon_{2} \sim$ $L^{-1}$ as usual. We have $\phi_{1}(1)=m_{\mathrm{s}}(L) \sim L^{0}$ but $\phi_{2}(1)$ behaves anomalously as $L^{-a+1 / 2}$. Finally, $x_{\mathrm{e}}^{\mathrm{s}}=a+1 / 2$ in the first order regime, $a>1 / 2$.

\section{Calculation of the moments}

In this section we evaluate the moments of the surface magnetization and the surface energy density at criticality on a chain with length $L$ in the regime of second-order surface transition. We restrict our study to the case of marginal perturbations, i.e., with $\kappa=1 / 2$ for model \#1 and $\kappa=1$ for model \#2.

\subsection{Surface magnetization}

We assume that the surface magnetization at $l=L-1$ on the dual chain, $\bar{m}_{\mathrm{s}}^{\mathrm{d}}(L)$ in $(15)$, is still scaling as $L^{-1 / 2}$ for the random models so that

$$
\left\langle m_{\mathrm{s}}^{q}(L)\right\rangle=L^{-q / 2}\left\langle\prod_{l=1}^{L-1} J_{l}^{q}\right\rangle=L^{-q / 2} \prod_{l=1}^{L-1}\left\langle J_{l}^{q}\right\rangle
$$

where we used the statistical independence of the couplings on different bonds. Using the distribution of the couplings given in (3) for model $\# 1$, with $J=1$ we obtain

$$
\left\langle J_{l}^{q}\right\rangle=\frac{1}{2}\left(1+\frac{a_{1}}{l^{1 / 2}}\right)^{q}+\frac{1}{2}\left(1-\frac{a_{1}}{l^{1 / 2}}\right)^{q} \simeq 1+\frac{q(q-1)}{2} \frac{a_{1}^{2}}{l} .
$$

The last expression gives the asymptotic behaviour when $l \gg 1$ which governs the critical behaviour. Taking the logarithm of the product in (20) and a 
continuum approximation leads to

$$
\ln \prod_{l=1}^{L-1}\left\langle J_{l}^{q}\right\rangle \simeq \int_{1}^{L} \ln \left(1+\frac{q(q-1)}{2} \frac{a_{1}^{2}}{l}\right) \mathrm{d} l \simeq \frac{q(q-1) a_{1}^{2}}{2} \ln L .
$$

Thus the $q$ th moment of the surface magnetization scale as

$$
\left\langle m_{\mathrm{s}}^{q}(L)\right\rangle \sim L^{-q x_{\mathrm{m}}^{\mathrm{s}}(q)} \sim L^{-(q / 2)\left[1-(q-1) a_{1}^{2}\right]}
$$

and the surface magnetization displays multiscaling with

$$
x_{\mathrm{m}}^{\mathrm{s}}(q)=\frac{1}{2}\left[1-(q-1) a_{1}^{2}\right] \quad(\text { model } \# 1) .
$$

Similarly for model \#2, using (4) with $J=1$ and $\kappa=1$, we obtain

$$
\left\langle J_{l}^{q}\right\rangle=\frac{1}{l}\left(1+a_{2}\right)^{q}+1-\frac{1}{l}=1+\frac{\left(1+a_{2}\right)^{q}-1}{l} .
$$

Since

$$
\ln \prod_{l=1}^{L-1}\left\langle J_{l}^{q}\right\rangle \simeq \int_{1}^{L} \ln \left[1+\frac{\left(1+a_{2}\right)^{q}-1}{l}\right] \mathrm{d} l \simeq\left[\left(1+a_{2}\right)^{q}-1\right] \ln L
$$

the $q$ th moment of the surface magnetization scales as

$$
\left\langle m_{\mathrm{s}}^{q}(L)\right\rangle \sim L^{-(q / 2)+\left(1+a_{2}\right)^{q}-1},
$$

so that

$$
x_{\mathrm{m}}^{\mathrm{s}}(q)=\frac{1}{2}-\frac{\left(1+a_{2}\right)^{q}-1}{q} \quad(\text { model \#2). }
$$

The exponents in Eqs. (24) and (28) are in agreement with the average and typical exponents obtained previously in Ref. [24] when $q=1$ and $q \rightarrow 0$, respectively:

$$
\begin{array}{lll}
{\left[x_{\mathrm{m}}^{\mathrm{s}}\right]_{\mathrm{av}}=\frac{1}{2},} & {\left[x_{\mathrm{m}}^{\mathrm{s}}\right]_{\mathrm{typ}}=\frac{1}{2}\left(1+a_{1}^{2}\right)} & (\text { model \#1) } \\
{\left[x_{\mathrm{m}}^{\mathrm{s}}\right]_{\mathrm{av}}=\frac{1}{2}-a_{2},} & {\left[x_{\mathrm{m}}^{\mathrm{s}}\right]_{\mathrm{typ}}=\frac{1}{2}-\ln \left(1+a_{2}\right)} & (\text { model \#2) }
\end{array}
$$




\subsection{Surface energy density}

For the scaling behaviour of the moments of the surface energy density, one can start from the expression giving $e_{\mathrm{S}}(L)$ in Eq. (19). The factor $L^{-1}$ is associated with the scaling of the excitations and reflects the isotropy of the bulk critical behaviour. It should not be modified with a random surface perturbation, hence we have

$$
\left\langle e_{\mathrm{s}}^{q}(L)\right\rangle \sim L^{-q}\left\langle m_{\mathrm{s}}^{2 q}(L)\right\rangle
$$

The $q$ th moment of the surface energy density depends on the moment of order $2 q$ of the surface magnetization.

For model \#1, making use of Eq. (23), we obtain

$$
\left\langle e_{\mathrm{S}}^{q}(L)\right\rangle \sim L^{-q x_{\mathrm{e}}^{\mathrm{s}}(q)} \sim L^{-q\left[2-(2 q-1) a_{1}^{2}\right]},
$$

so that

$$
x_{\mathrm{e}}^{\mathrm{s}}(q)=2-(2 q-1) a_{1}^{2} \quad(\text { model } \# 1) .
$$

In the same way Eq. (27) leads to

$$
\left\langle e_{\mathrm{S}}^{q}(L)\right\rangle \sim L^{-2 q+\left(1+a_{2}\right)^{2 q}-1}
$$

for model \#2 so that

$$
x_{\mathrm{e}}^{\mathrm{s}}(q)=2-\frac{\left(1+a_{2}\right)^{2 q}-1}{q} \quad(\text { model \#2). }
$$

The average and typical exponents are then given by:

$$
\begin{array}{lll}
{\left[x_{\mathrm{e}}^{\mathrm{s}}\right]_{\mathrm{av}}=2-a_{1}^{2},} & {\left[x_{\mathrm{e}}^{\mathrm{s}}\right]_{\mathrm{typ}}=2+a_{1}^{2}} & (\text { model \#1) } \\
{\left[x_{\mathrm{e}}^{\mathrm{s}}\right]_{\mathrm{av}}=2-a_{2}\left(2+a_{2}\right),} & {\left[x_{\mathrm{e}}^{\mathrm{s}}\right]_{\mathrm{typ}}=2\left[1-\ln \left(1+a_{2}\right)\right]} & (\text { model \#2) }
\end{array}
$$

The typical exponents in Eqs. (29) and (35) are in agreement with the values conjectured in Ref. [24]. They keep the same form as for the pure HvL model in Eqs. (18) and (19) with a replaced by an effective amplitude, $-a_{1}^{2} / 2$ for model \#1 and $\ln \left(1+a_{2}\right)$ for model \#2. These typical effective amplitudes are deduced from the relation $\ln J_{l}\left(a_{\text {eff }}\right) \simeq\left\langle\ln J_{l}\right\rangle$ for $l \gg 1$. A similar conjecture proposed in Ref. [24] for the average exponents is actually verified only up to the first order in the perturbation amplitude. 


\section{Numerical results}

\subsection{Surface magnetization}

The surface magnetization for a given configuration of the couplings $\left\{J_{k}\right\}$ was obtained using Eq. (14) at criticality, i.e., with $h_{k}=1$ and $J=1$ in (3) and (4).

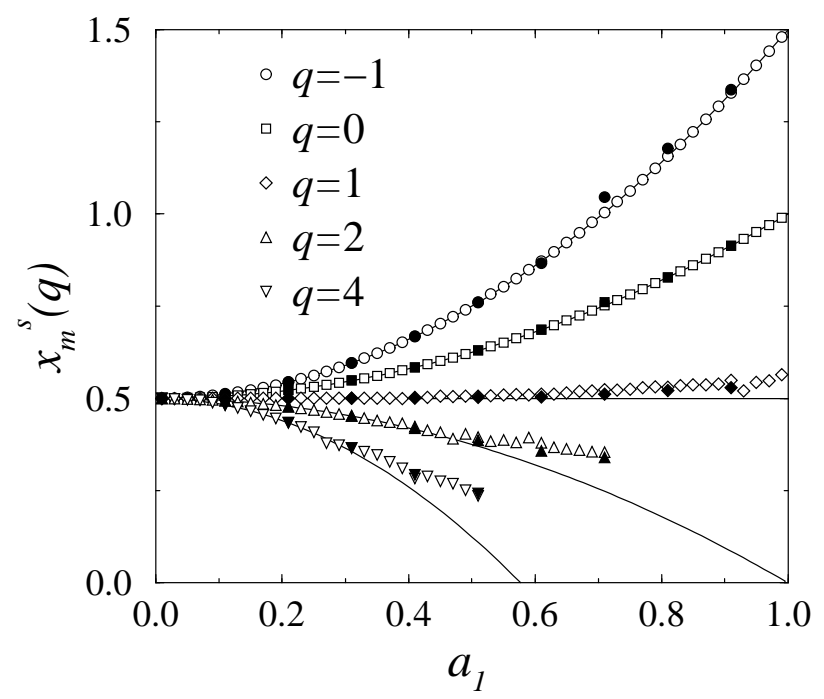

Fig. 1. Exponent of the $q$ th moment of the surface magnetization as a function of the perturbation amplitude for model \#1. The extrapolated exponents were deduced either from exact enumerations for chains with sizes $L=4-24$ (open symbols) or from Monte Carlo samplings for chains with sizes $L=2^{5}-2^{14}$ (filled symbols). The lines correspond to the analytical expression in (24).

The moments were averaged either by performing exact enumerations of the random configurations in the case of chains with lengths $L=4-24$ or through Monte Carlo samplings (over $10^{6}$ samples) for longer chains with lengths $L=2^{5}-2^{14}$. The exponents $x_{\mathrm{m}}^{\mathrm{s}}(q)$ were deduced from an extrapolation of twopoint approximants using the BST algorithm [31]. The marginal exponents are shown in Fig. 1 for model \#1 and in Fig. 2 for model \#2.

\subsection{Surface energy density}

The surface energy density was obtained using the expression given in (17) where $\epsilon_{1}, \epsilon_{2}, \phi_{1}(1)$ and $\phi_{2}(1)$ are obtained through the diagonalisation of a $L \times L$ matrix constructed from the elements of $\mathrm{T}^{2}$ with odd indices. 


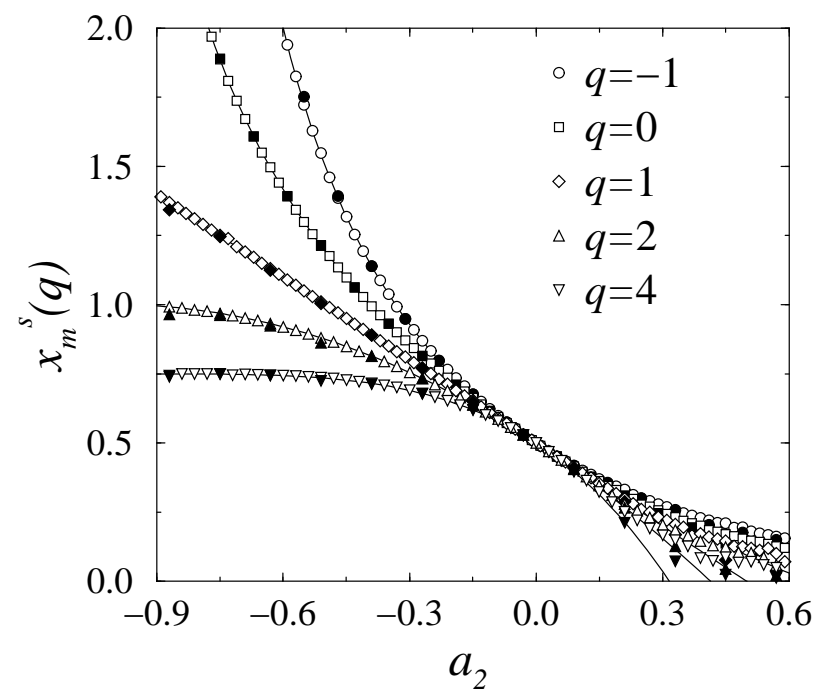

Fig. 2. As in Fig. 1 for model \#2. The lines correspond to the analytical expression in $(28)$.

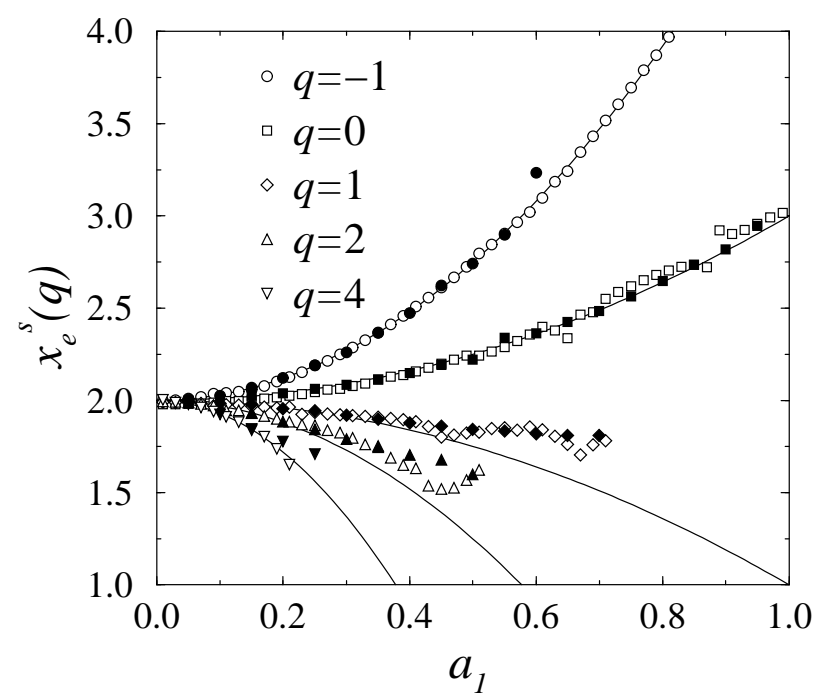

Fig. 3. Exponent of the $q$ th moment of the surface energy density as a function of the perturbation amplitude for model \#1. The extrapolated exponents were deduced either from exact enumerations for chains with sizes $L=4-24$ (open symbols) or from Monte Carlo samplings for chains with sizes $L=2^{2}-2^{9}$ (filled symbols). The lines correspond to the analytical expression in (32).

The average moments were deduced from exact enumerations for chains with lengths $L=4-24$ and from Monte Carlo samplings (over $10^{4}$ samples) for chains with lengths $L=2^{2}-2^{9}$. The exponents $x_{\mathrm{e}}^{\mathrm{s}}(q)$ were deduced from an extrapolation of two-point approximants using the BST algorithm in the case of exact enumerations. The approximants deduced from the Monte Carlo data were too noisy to use the BST extrapolation process. In this case the exponents were deduced from a non-linear fit of the following expression:

$$
\ln \left\langle e_{\mathrm{s}}^{q}(L)\right\rangle \simeq \ln A-x_{\mathrm{e}}^{\mathrm{s}}(q) \ln L+B L^{-\omega} .
$$




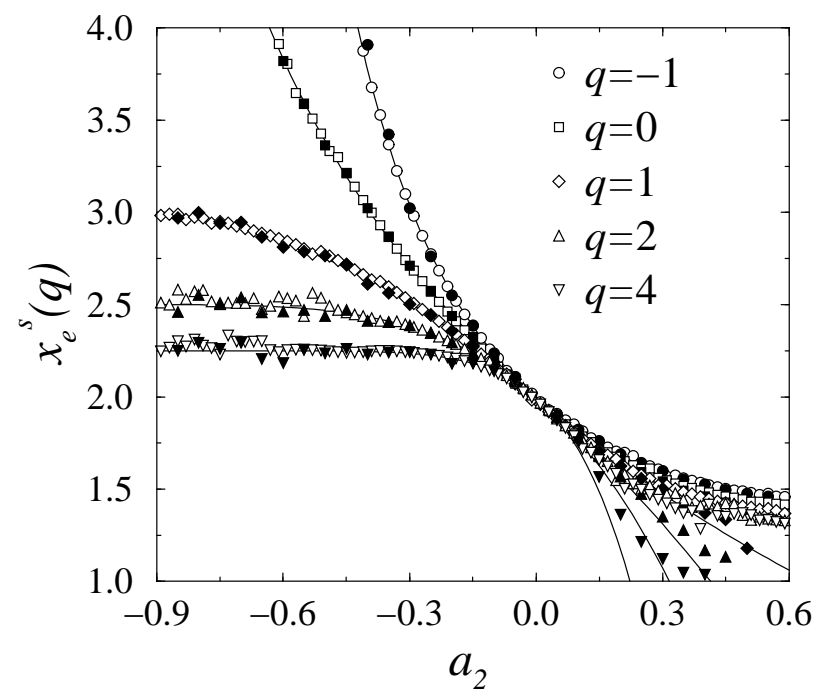

Fig. 4. As in Fig. 3 for model \#2. The lines correspond to the analytical expression in $(34)$.

The continuously varying exponents are compared to the analytical expressions in Fig. 3 for model \#1 and in Fig. 4 for model \#2.

\section{Discussion}

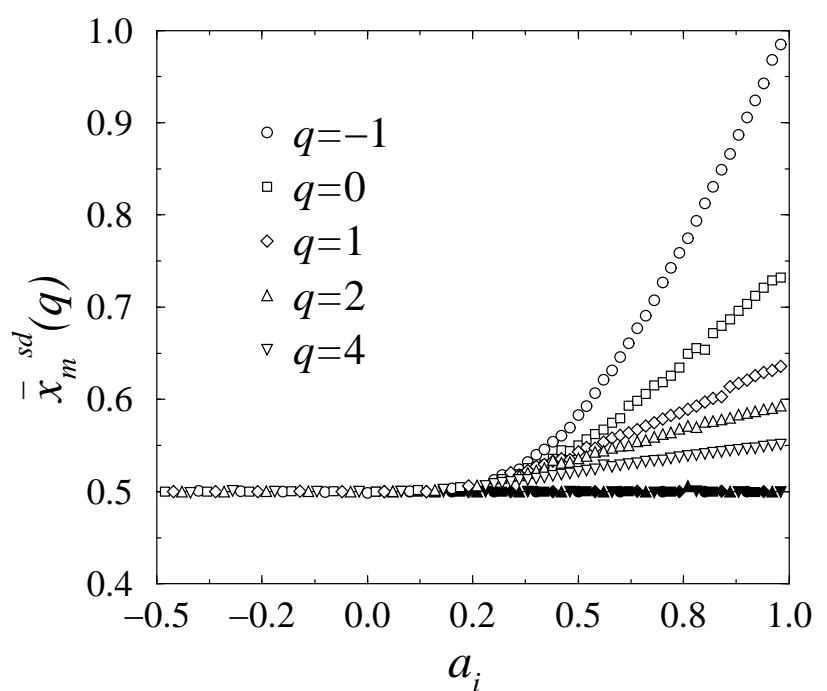

Fig. 5. Exponent of the $q$ th moment of the dual surface magnetization at $L$ as a function of the perturbation amplitude for model \#1 (full symbols) and model \#2 (open symbols). The extrapolated exponents were deduced from exact enumerations for chains with sizes $L=4-24$. The line corresponds to the behaviour at the ordinary surface transition where $x_{\mathrm{m}}^{\mathrm{s}}=1 / 2$.

A good agreement between numerical and conjectured analytical results have 
been obtained for the surface magnetization and the surface energy density, except in the vicinity of first-order surface transitions.

Already for the pure HvL model a slow convergence to the exactly known behaviour was observed close to the first-order regime [13]. One may verify on Figs. 2 and 4 for model \#2 that the Monte Carlo data, obtained on larger systems, are closer to the analytical results when $a_{2}>0$. Nevertheless, like for the the pure system, some of the scaling assumptions leading to the analytical expressions for the surface exponents cannot be valid beyond some critical value of the perturbation amplitude since $x_{\mathrm{m}}^{\mathrm{s}}(q)$ and $x_{\mathrm{e}}^{\mathrm{s}}(q)$ have to remain non-negative.

The dual magnetization in (15) was assumed to scale as $L^{-1 / 2}$, like for the unperturbed system at the ordinary transition. We performed a numerical study of the moments of $\bar{m}_{\mathrm{s}}^{\mathrm{d}}(L)$ for the two models using Eq. (16), exact enumerations for chains with lengths $L=4-24$ and BST extrapolations. The results are shown in Fig. 5.

For model \#1, the ordinary surface behaviour is obtained for any value of the perturbation amplitude which gives some confidence in the conjectured analytical expressions in the regime of second-order transition. For model \#2, the ordinary surface behaviour is only obtained when $a_{2} \lesssim .2$. Beyond this value deviations from the ordinary surface behaviour are observed and the analytical expressions for the exponents are probably no longer valid.

\section{Acknowledgements}

Useful discussions with Dragi Karevski and Ferenc Iglói are gratefully acknowledged.

\section{References}

[1] H.J. Hilhorst, J.M.J. van Leeuwen, Phys. Rev. Lett. 47 (1981) 1188.

[2] R. Cordery, Phys. Rev. Lett. 48 (1982) 215.

[3] T.W. Burkhardt, Phys. Rev. Lett. 48 (1982) 216.

[4] T.W. Burkhardt, Phys. Rev. B 25 (1982) 7048.

[5] I. Peschel, Phys. Rev. B 30 (1984) 6783.

[6] H.W.J. Blöte, H.J. Hilhorst, Phys. Rev. Lett. 51 (1983) 20. 
[7] T.W. Burkhardt, I. Guim, Phys. Rev. B 29 (1984) 508.

[8] T.W. Burkhardt, I. Guim, H.J. Hilhorst, J.M.J. van Leeuwen, Phys. Rev. B 30 (1984) 1486.

[9] H.W.J. Blöte, H.J. Hilhorst, J. Phys. A 18 (1985) 3039.

[10] R.Z. Bariev, L. Turban, Phys. Rev. B 45 (1992) 10761.

[11] F. Iglói, L. Turban, Phys. Rev. B 47 (1993) 3404.

[12] T.W. Burkhardt, F. Iglói, J. Phys. A 23 (1990) L633.

[13] B. Berche, L. Turban, J. Phys. A 23 (1990) 3029.

[14] L. Turban, B. Berche, J. Phys. A 26 (1993) 3131.

[15] D. Karevski, L. Turban, F. Iglói, J. Phys. A 33 (2000) 2663.

[16] R.Z. Bariev, Sov. Phys. JETP 67 (1988) 2170.

[17] R.Z. Bariev, O.A. Malov, Phys. Lett. A 136 (1989) 291.

[18] F. Iglói, B. Berche, L. Turban, Phys. Rev. Lett. 65 (1990) 1773.

[19] L. Turban, Phys. Rev. B 44 (1991) 7051.

[20] R.Z. Bariev, I. Peschel, J. Phys. A 24 (1991) L87.

[21] I. Peschel, R. Wunderling, Ann. Phys. (Leipzig) 1 (1992) 125.

[22] F. Iglói, I. Peschel, L. Turban, Adv. Phys. 42 (1993) 683.

[23] L. Turban, Eur. Phys. J. B 11 (1999) 273.

[24] L. Turban, D. Karevski, F. Iglói, J. Phys. A 32 (1999) 3907.

[25] D. Karevski, R. Juhász, L. Turban, F. Iglói, Phys. Rev. B 60 (1999) 4195.

[26] J. Kogut, Rev. Mod. Phys. 51 (1979) 659.

[27] E.H. Lieb, T.D. Schultz, D.C. Mattis, Ann. Phys. (N.Y.) 16 (1961) 406.

[28] P. Pfeuty, Ann. Phys. (Paris) 57 (1970) 79.

[29] P. Jordan, E. Wigner, Z. Phys. 47 (1928) 631.

[30] P.E. Berche, B. Berche, L. Turban, J. Phys. I (France) 6 (1996) 621.

[31] M. Henkel, G. Schütz, J. Phys. A 21 (1988) 2617. 